\title{
ANALYTIC EXTENSIONS OF ALGEBRAIC ISOMORPHISMS
}

\author{
S. KALIMAN
}

(Communicated by Harm Derksen)

\begin{abstract}
Let $\Psi: X_{1} \rightarrow X_{2}$ be an isomorphism of closed affine algebraic subvarieties of $\mathbb{C}^{n}$ such that $n>\max \left(2 \operatorname{dim} X_{1}, \operatorname{dim} T X_{1}\right)$. We prove that $\Psi$ can be extended to a holomorphic automorphism of $\mathbb{C}^{n}$. Furthermore, when $\Psi$ is an isomorphism of curves, such an extension exists for every $n \geq 3$ even when $\operatorname{dim} T X_{1}=n$.
\end{abstract}

\section{INTRODUCTION}

Let $\Psi: X_{1} \rightarrow X_{2}$ be an isomorphism of closed affine algebraic subvarieties of $\mathbb{C}^{n}$. It was proven in [8], 10] that $\Psi$ is the restriction of an algebraic automorphism of $\mathbb{C}^{n}$ provided $n>\max \left(2 \operatorname{dim} X_{1}+1, \operatorname{dim} T X_{1}\right)$. The inequality on $\operatorname{dim} T X_{1}$ cannot be improved [8] while the question whether $2 \operatorname{dim} X_{1}+1$ is the optimal bound in this theorem remains open. It is unknown, for instance, whether an isomorphism of two closed curves in $\mathbb{C}^{3}$ (even when these curves are isomorphic to $\mathbb{C}$ ) can be extended to an algebraic automorphism of $\mathbb{C}^{3}$. However, any two algebrai 1 embeddings of $\mathbb{C}$ into $\mathbb{C}^{3}$ are equivalent up to a holomorphic coordinate substitution [9]. In this paper we get the following improvements of the last statement.

Theorem 0.1. Let $\Psi: X_{1} \rightarrow X_{2}$ be an isomorphism of closed affine algebraic subvarieties of $\mathbb{C}^{n}$ and $n>\max \left(2 \operatorname{dim} X_{1}\right.$, $\left.\operatorname{dim} T X_{1}\right)$. Then $\Psi$ can be extended to a holomorphic automorphism $\tilde{\Psi}$ of $\mathbb{C}^{n}$.

In particular, we have the following:

(a) different closed embeddings of an affine algebraic variety $X$ into $\mathbb{C}^{n}$ with $n>$ $\max (2 \operatorname{dim} X, \operatorname{dim} T X)$ can be moved to each other by a holomorphic automorphism of $\mathbb{C}^{n}$;

(b) under similar dimension assumptions every algebraic automorphism of a closed affine algebraic subvariety of $\mathbb{C}^{n}$ can be extended to a holomorphic automorphism of $\mathbb{C}^{n}$.

It is worth mentioning that facts analogous to (b) are not automatic (e.g., see [2]).

Received by the editors September 15, 2013 and, in revised form, October 26, 2013.

2010 Mathematics Subject Classification. Primary 14R20, 32M17.

Key words and phrases. Affine varieties, group actions, one-parameter subgroups, transitivity.

This research was partially done during a visit of the author to the Max-Planck-Institute of Mathematics, Bonn. He thanks this institution for generous support and excellent working conditions.

${ }^{1}$ Precaution: the similar statement does not hold for proper holomorphic embeddings of $\mathbb{C}$ into $\mathbb{C}^{n}$ for any $n \geq 2$; see [6], 9 . 
Theorem 0.2. Let $X_{1}$ and $X_{2}$ be closed affine algebraic curves in $\mathbb{C}^{n}, n \geq 3$, and let $\Psi: X_{1} \rightarrow X_{2}$ be an isomorphism. Then $\Psi$ extends to a holomorphic automorphism of $\mathbb{C}^{n}$.

That is, in the case of an isomorphism of closed curves in $\mathbb{C}^{3}$ there are no analytic invariants that can prevent an extension to an algebraic automorphism.

\section{Semi-ISOMORPHisms}

Our central technical tools will be the following notion of semi-isomorphism and also a weaker notion of pseudo-isomorphism that will be used in Section 3 .

Definition 1.1. Let $\varphi: X \rightarrow Y$ be a holomorphic map of Stein spaces. We call $\varphi$ a semi-isomorphism (resp. pseudo-isomorphism) if the following conditions hold:

(a) $\varphi$ is a finite bimeromorphic map;

(b) for every $x \in X$ (resp. every smooth point $x \in X$ ) there exists a neighborhood $U \subset X$ of $x$ such that $\left.\varphi\right|_{U}$ is an embedding;

(c) there exists a neighborhood $U_{0}$ of the singular set $X_{\text {sing }}$ of $X$ such that $U_{0}=\varphi^{-1}\left(\varphi\left(U_{0}\right)\right)$ and the restriction of $\left.\varphi\right|_{U_{0}}: U_{0} \rightarrow \varphi\left(U_{0}\right)$ is an embedding (resp. injection), while the singular set $Y_{\text {sing }}$ of $Y$ is a disjoint union of $\varphi\left(X_{\text {sing }}\right)$ and a discrete set of nodes (i.e. singular points $y \in Y$ such that the analytic germ of $Y$ at $y$ is a union of two smooth irreducible branches meeting transversally at $y$ ).

We apply these notions mostly in the algebraic case when $\varphi: X \rightarrow Y$ is a morphism of affine algebraic varieties over $\mathbb{C}$ (which will be the ground field throughout the paper). The main property of semi-isomorphisms we are going to exploit is given by the next straightforward fact.

Proposition 1.2. Let $\varphi: X \rightarrow Y$ be a semi-isomorphism as in Definition 1.1. Let $y_{1}, y_{2}, \ldots$ be the set of nodes of $Y$ not contained in $\varphi\left(X_{\text {sing }}\right)$. Suppose that $\varphi^{-1}\left(y_{i}\right)=\left\{x_{i}^{\prime}, x_{i}^{\prime \prime}\right\}$ for every $i$. Then the algebra $\mathcal{O}(Y)$ of holomorphic functions on $Y$ is naturally isomorphic to the subalgebra $R$ of the algebra $\mathcal{O}(X)$ of holomorphic functions on $X$ such that $f \in R$ if and only if $f\left(x_{i}^{\prime}\right)=f\left(x_{i}^{\prime \prime}\right)$ for every $i=1, \ldots, k$.

To describe semi-isomorphisms geometrically in the algebraic situation we need the following.

Notation 1.3. For $m \leq n$ the space of linear surjective maps (projections) from $\mathbb{C}^{n}$ to $\mathbb{C}^{m}$ will be denoted by $L_{n, m}$. This notion depends on the choice of coordinate system in $\mathbb{C}^{n}$, i.e. on the choice of an embedding of $\mathbb{C}^{n}$ into $\mathbb{P}^{n}=\mathbb{C}^{n} \cup H$ where $H$ is the hyperplane at infinity. For every affine subvariety $X$ of $\mathbb{C}^{n}$, denote by $\bar{X}$ its closure in $\mathbb{P}^{n}$ and by $X_{H}$ the variety $\bar{X} \cap H$. For every $x \in X$ one can treat the Zariski tangent space $T_{x} X$ as a linear subspace of $\mathbb{C}^{n}$. With this interpretation we consider the variety $T^{\prime} X=\left\{x+v \mid x \in X, v \in T_{x} X\right\}$ in $\mathbb{C}^{n}$. We let $C h(X)$ be the variety of chords of $X$ (recall that a chord is a line that passes through at least two points of $X$ ) and let $C X$ be the closure (in $\mathbb{C}^{n}$ ) of $\bigcup_{\ell \in C h(X)} \ell$. Note that there is a natural morphism $\pi: C h(X) \rightarrow(C X)_{H}$ such that for general $z \in(C X)_{H}$ the preimage $\pi^{-1}(z)$ consists of parallel chords of $X$ whose direction is determined by $z$.

We need also the following.

Lemma 1.4. Let $X$ be a closed proper affine algebraic subvariety of $\mathbb{C}^{n}$ such that $n \geq 2 \operatorname{dim} X+1$. Then the coordinate system in $\mathbb{C}^{n}$ can be chosen so that for any 
pair of general points $x_{1}, x_{2}$ in $X$ (not necessarily in the same irreducible component 2 the following condition holds

the vector space generated by $T_{x_{1}} X, T_{x_{2}} X$ and the vector $\overrightarrow{x_{1}, x_{2}}$ has dimension $\operatorname{dim} T_{x_{1}} X+\operatorname{dim} T_{x_{2}} X+1$ (in particular, it is $2 \operatorname{dim} X+1$ when $x_{1}$ and $x_{2}$ belong to components of $X$ with the largest dimension).

Proof. Let $A$ be a finite set of general points of $X$ such that every irreducible component of $X$ contains at least two points from $A$. By [1, Theorem 4.14 and Remark 4.16] there exists an algebraic automorphism $\beta$ of $\mathbb{C}^{n}$ that fixes every point $a \in A$ and has prescribed values of $\left.(\beta)_{*}\right|_{T_{a} \mathbb{C}^{n}}$ in $S L\left(T_{a} \mathbb{C}^{n}\right)$ for each $a \in A$. Choosing an appropriate collection $\left\{\left.(\beta)_{*}\right|_{T_{a} \mathbb{C}^{n}} \mid a \in A\right\}$ and replacing $X$ by $\beta(X)$ we get condition (\#).

Remark 1.5. (1) Let $\varphi \in L_{n, n-1}$ be such that $\overrightarrow{x_{1}, x_{2}} \in \operatorname{Ker} \varphi$ for $x_{1}, x_{2}$ as in Lemma 1.4. Then condition (\#) implies that $\varphi\left(T_{x_{1}} X\right) \cap \varphi\left(T_{x_{2}} X\right)=\{0\}$.

(2) One can apply [1, Theorem 4.14 and Remark 4.16] in the proof of Lemma 1.4 because $\mathbb{C}^{n}$ is the so-called flexible variety. Let $Y$ be a closed subvariety of $\mathbb{C}^{n}$ with $\operatorname{dim} Y \leq n-2$. Then $\mathbb{C}^{n} \backslash Y$ is still flexible (e.g., see [4]). In particular, if $Y$ does not contain any irreducible component of $X$, then the automorphism of $\mathbb{C}^{n}$ that induces a new coordinate system in Lemma 1.4 can be chosen so that its restriction to $Y$ (and even to any given infinitesimal neighborhood of $Y$ ) is identical [4, Theorem 1.6].

(3) A similar proof shows that Lemma 1.4 is valid in the case when $X$ is a closed proper analytic subset of $\mathbb{C}^{n}$ with a finite number of irreducible components. A bit more complicated argument implies that it remains true even for an infinite number of components (but we shall not need this fact later).

Convention 1.6. Further in this section we suppose that $X$ is a closed proper affine algebraic subvariety of $\mathbb{C}^{n}$ such that $n \geq 2 \operatorname{dim} X+1$ and $\operatorname{dim} T X \leq 2 \operatorname{dim} X$ where $T X$ is the Zariski tangent bundle of $X$. We continue to treat every tangent space $T_{x} X$ as a natural subspace of $\mathbb{C}^{n}$ and suppose that condition (\#) of Lemma 1.4 holds.

Lemma 1.7. Let Convention 1.6 hold. Then $\operatorname{dim} C X=2 \operatorname{dim} X+1$ and therefore $\operatorname{dim}(C X)_{H}=2 \operatorname{dim} X$. Furthermore, there is a subvariety $(C X)_{H}^{\prime} \subset(C X)_{H}$ of dimension at most $2 \operatorname{dim} X-1$ such that for every $z \in(C X)_{H} \backslash(C X)_{H}^{\prime}$

(i) the preimage $\pi^{-1}(z)$ is at most finite;

(ii) for each $\ell \in \pi^{-1}(z)$ every pair of distinct points $x_{1}$ and $x_{2}$ in $\ell \cap X$ satisfies condition (\#): $: 3$

(iii) $\ell$ does not contain points from $X_{\text {sing }}$ and $\ell$ is not tangent to $X$ at any smooth point;

(iv) $\ell$ meets $X$ at exactly two points.

Proof. Consider general points $x_{1}$ and $x_{2}$ in an irreducible component $D$ of $X$ such that $\operatorname{dim} D=\operatorname{dim} X$. Let $U_{i}$ be a small Euclidean neighborhood of $x_{i}$ in $D$, i.e. $U_{i}$

\footnotetext{
${ }^{2}$ Recall that a point of an algebraic variety $X$ is called general if it is not contained in a proper closed subvariety $Z$ of $X$. We require additionally that such $Z$ does not contain any irreducible component of $X$.

${ }^{3}$ That is, in the case of $n=2 \operatorname{dim} X+1$ (i.e., $\operatorname{dim} H=2 \operatorname{dim} X$ ) for general $\varphi \in L_{n, n-1}$ and every pair of points $x_{1}, x_{2} \in X$ such that $\overrightarrow{x_{1}, x_{2}} \in \operatorname{Ker} \varphi$ one has condition (\#).
} 
corresponds to a neighborhood of the origin in $T_{x_{i}} D$. Condition (\#) implies that the union of chords through points of $U_{1}$ and $U_{2}$ is of dimension $2 \operatorname{dim} X+1$ which yields the first statement.

Consider the subvariety $S \subset X$ that includes $X_{\text {sing }}$ and all points $x_{1} \in X$ such that for every $x_{2} \in X$ condition (\#) does not hold. We can suppose that $\operatorname{dim} S \leq \operatorname{dim} X-1$. The union $B$ of chords containing points from $S$ has dimension at most $\operatorname{dim} S+\operatorname{dim} X+1 \leq 2 \operatorname{dim} X$. That is, $\operatorname{dim} B_{H}<\operatorname{dim} H$. Similarly the union $P$ of the chords containing pairs $\left(x_{1}, x_{2}\right)$ of distinct points of $X_{\text {reg }}$, that do not satisfy condition (\#), is of dimension at most $2 \operatorname{dim} X$ (since for a fixed $x_{1}$ the variety of all $x_{2} \in X$, for which (\#) is invalid, is of dimension at most $\operatorname{dim} X-1$ ). Hence $\operatorname{dim} P_{H}<\operatorname{dim} H$. Note also that by Convention 1.6 $\operatorname{dim}\left(T^{\prime} X\right)_{H}<2 \operatorname{dim} X$. Choosing $(C X)_{H}^{\prime}$ so that $B_{H}, P_{H}$, and $\left(T^{\prime} X\right)_{H}$ are contained in $(C X)_{H}^{\prime}$ we get (ii) and (iii). In particular every $\ell$ is a line that meets $X$ transversally at the intersection points.

Suppose that $\pi^{-1}(z)$ is not finite. That is, assuming that $x_{1}, x_{2} \in \ell$ and $U_{1}, U_{2}$ are as before we can find biholomorphic analytic subsets $W_{i} \subset U_{i}$ of positive dimensions such that every $w_{1} \in W_{1}$ and its image $w_{2}$ in $W_{2}$ are joined by a chord from $\pi^{-1}(z)$. This implies that for $\varphi$ from Remark 1.5 (1) $\varphi\left(T_{x_{1}} X\right) \cap \varphi\left(T_{x_{2}} X\right)$ contains $\varphi\left(T_{x_{1}} W_{1}\right) \simeq \varphi\left(T_{x_{2}} W_{2}\right)$. Hence condition (\#) does not hold by Remark 1.5 (1) which contradicts (ii). That is, $W_{i}$ is zero-dimensional and we have (i).

Note that the number of points in $\ell \cap X$ is constant for general $z \in(C X)_{H}$ and these points depend continuously on $z$. (Indeed, there is a natural morphism from the complement to the diagonal in $X \times X$ into $(C X)_{H}$ and the statement is a consequence of the semi-continuity theorem [7.) Assume that $\ell$ contains at least three points $x_{1}, x_{2}$ and $x_{3}$ in $X$, i.e. the vectors $\overrightarrow{x_{1} x_{2}}$ and $\overrightarrow{x_{1} x_{3}}$ are parallel. Let $U_{i}$ be a neighborhood $x_{i}$ as before. Choose a sequence of points $x_{2 i}$ in $U_{2}$ convergent to $x_{2}$ and consider the chords through $x_{1}$ and $x_{2 i}$. By semi-continuity and continuous dependence on $z$ there must be a point $x_{3 i} \in U_{3}$ on the same chord as $x_{1}$ and $x_{2 i}$. However this implies that for $\psi \in L_{n, n-1}$ with $\ell \in \operatorname{Ker} \psi$ one would have again $\psi\left(T_{x_{2}} X\right) \cap \psi\left(T_{x_{3}} X\right) \neq\{0\}$ contrary to condition (\#). Hence we have (iv).

The following is the main result of this section.

Theorem 1.8. Let $X$ be a closed affine subvariety of $\mathbb{C}^{n}$ with Convention 1.6 valid, and let $2 \operatorname{dim} X \leq m<n$. Suppose that $\varphi$ is a general element of $L_{n, m}$. Then $\left.\varphi\right|_{X}: X \rightarrow Y:=\varphi(X) \subset \mathbb{C}^{m}$ is a semi-isomorphism.

Proof. Since for $m=2 \operatorname{dim} X+1$ and general $\varphi \in L_{n, m}$ the map $\left.\varphi\right|_{X}: X \rightarrow Y$ is an isomorphism onto a closed subvariety of $\mathbb{C}^{m}$ (e.g., see [8]) we can suppose that $n=2 \operatorname{dim} X+1$ and $m=2 \operatorname{dim} X$. Then by Lemma 1.7 (i) $\left.\varphi\right|_{X}: X \rightarrow Y$ is bijective in the complement to a finite set for general $\varphi$. That is, such a $\varphi$ is birational.

Since $V=\operatorname{Ker} \varphi$ is now a line, $V_{H}$ is a general point in $H$. Then $V_{H} \cap X_{H}=\varnothing$ because $\operatorname{dim} X_{H}=\operatorname{dim} X-1$. Hence $\left.\varphi\right|_{X}: X \rightarrow Y$ is a proper morphism, and $Y$ is closed in $\mathbb{C}^{m}$. Furthermore being proper and quasi-finite, $\left.\varphi\right|_{X}$ is finite by Grothendieck's theorem.

Similarly $V_{H} \cap\left(T^{\prime} X\right)_{H}=\varnothing$ for a general $\varphi$ since $\operatorname{dim} T X \leq 2 \operatorname{dim} X$ and thus $\operatorname{dim}\left(T^{\prime} X\right)_{H} \leq 2 \operatorname{dim} X-1$. Hence for every $x \in X$ the linear map $\varphi_{*}: T_{x} X^{\prime} \rightarrow$ $T_{\varphi(x)} Y^{\prime}$ is an isomorphism where $X^{\prime}$ is the analytic germ of $X$ at $x$ and $Y^{\prime}=\varphi\left(X^{\prime}\right)$. 
This implies that for some neighborhood $U \subset X$ of $x$ the restriction $\left.\varphi\right|_{U}$ is an embedding (e.g, see [8, Proposition 7]).

Thus we have conditions (a) and (b) from Definition 1.1 while condition (c) follows from Lemma 1.7 (ii), (iii), and (iv).

Remark 1.9. (1) Since $\varphi \in L_{n, m}$ in Theorem 1.8 is general we can suppose that any forgetting projection $\mathbb{C}^{n} \rightarrow \mathbb{C}^{m}$ to a coordinate subspace of dimension $m$ can serve as $\varphi$ in this theorem.

(2) Since any semi-isomorphism does not distinguish only a finite number of points and any general linear function separates elements of finite sets, we can also suppose that any forgetting projection $p: \mathbb{C}^{n} \rightarrow \mathbb{C}^{m+1}$ yields an isomorphism between $X$ and $p(X)$.

Furthermore, one can see that the proof yields a stronger statement.

Proposition 1.10. Let $X$ and $Y$ be as in Theorem 1.8. Let $l=2 \operatorname{dim} X$ and $M$ be an irreducible subvariety of $L_{n, l}$ such that the collection $\{\operatorname{Ker} \varphi \mid \varphi \in M\}$ generates a subvariety of dimension at least $l+1$ in the Grassmannian $\operatorname{Gr}(n, n-l)$. Suppose also that for any pair of general points $x_{1}, x_{2} \in X$ there exists $\varphi \in M$ with $\varphi\left(x_{1}\right)=\varphi\left(x_{2}\right)$ and valid condition (\#) from Lemma 1.4, Then the restriction $\left.\varphi\right|_{X}: X \rightarrow Y$ is a semi-isomorphism.

Proof. Indeed, for $1 \leq m \leq n$ and every $\psi \in L_{n, m}$ let us treat $\psi\left(\mathbb{C}^{n}\right)$ as the orthogonal complement to $\operatorname{Ker} \psi$ (i.e. as a subspace of $\mathbb{C}^{n}$ ). Then one can fix a general $\psi \in L_{n, l+1}$ such that $\left.\psi\right|_{X}: X \rightarrow \psi(X)$ is an isomorphism onto the closed subvariety $\psi(X)$ of $\mathbb{C}^{l+1}$ and for general $\varphi \in M$ the restriction of $\psi$ to $\varphi\left(\mathbb{C}^{n}\right) \subset \mathbb{C}^{n}$ is injective. We can present now $\psi \circ \varphi: \mathbb{C}^{n} \rightarrow \mathbb{C}^{l}$ as a composition $\varphi^{\prime} \circ \psi: \mathbb{C}^{n} \rightarrow \mathbb{C}^{l}$ where $\varphi^{\prime} \in L_{l+1, l}$, and replace $X$ by $\psi(X)$ and let $M$ be the family $M^{\prime}$ that consists of such $\varphi^{\prime}$. Generality of $\psi$ implies that its restriction to the subspace generated by $T_{x_{1}} X, T_{x_{2}} X$ and the vector $\overrightarrow{x_{1}, x_{2}}$ is injective and therefore the modified condition (\#) does not suffer under this change from $M$ to $M^{\prime}$. Furthermore, the assumption on dimension implies that the set $\left\{\operatorname{Ker} \varphi^{\prime} \mid \varphi^{\prime} \in M^{\prime}\right\}$ is dense in $\operatorname{Gr}(l+1,1) \simeq \mathbb{P}^{l}$. Treating the last variety as $H \subset \mathbb{P}^{l+1}$ we get the desired conclusion from Theorem 1.8 .

To describe a specific submanifold $M$ needed later we introduce the following.

Notation 1.11. Let $\Psi: X_{1} \rightarrow X_{2}$ be an isomorphism of closed affine algebraic subvarieties of $\mathbb{C}^{n}$ where $n=2 \operatorname{dim} X_{1}+1$. Suppose that Convention 1.6 is true for the embeddings $X_{i} \subset \mathbb{C}^{n}, i=1,2$, and the restriction of forgetting projections satisfy Remark 1.9 (1). Consider the graph $\Gamma$ of $\Psi$ in $\mathbb{C}^{2 n}$ with natural coordinates $(\bar{z}, \bar{w})=\left(z_{1}, \ldots, z_{n}, w_{1}, \ldots, w_{n}\right)$ and the forgetting projections $\varrho_{m, k}: \mathbb{C}^{2 n} \rightarrow \mathbb{C}^{m+k}$ given by

$$
(\bar{z}, \bar{w}) \rightarrow\left(z_{1}, \ldots, z_{m}, w_{1}, \ldots, w_{k}\right) .
$$

Proposition 1.12. Let Notation 1.11 hold and $l=2 \operatorname{dim} X_{1}$. Suppose that $M$ is the subvariety of $L_{2 n, l}$ that consists of all projections of form $\varphi=\left(\varphi_{1}, \varphi_{2}\right)$ where $\varphi_{1} \in L_{n, m}$ depends on $\bar{z}$ only, while $\varphi_{2} \in L_{n, l-m}$ depends on $\bar{w}$ only. Then there is a polynomial coordinate substitution in $\mathbb{C}^{2 n}=\mathbb{C}^{n} \oplus \mathbb{C}^{n}$ of form $\alpha=\left(\alpha_{1}, \alpha_{2}\right)$ where $\alpha_{i}$ is an algebraic automorphism of $\mathbb{C}^{n}$ such that after this substitution the restriction $\left.\varphi\right|_{\Gamma}: \Gamma \rightarrow \varphi(\Gamma)$ is a semi-isomorphism onto a closed subvariety of $\mathbb{C}^{l}$ for any general $\varphi \in M$. In particular, one can assume (by Remark 1.9) that the 
restriction to $\Gamma$ of each $\varrho_{m, l-m}$ is a semi-isomorphism and of each $\varrho_{m, n-m}$ (or $\left.\varrho_{m+1, l-m}\right)$ is an isomorphism.

Proof. Consider general points $a_{1}$ and $a_{2}$ in $\Gamma$. Suppose that $\varphi \in M$ is such that $\varphi\left(a_{1}\right)=\varphi\left(a_{2}\right)$. Let $\varphi=\left(\varphi_{1}, \varphi_{2}\right): \mathbb{C}^{2 n}=\mathbb{C}^{n} \oplus \mathbb{C}^{n} \rightarrow \mathbb{C}^{l}=\mathbb{C}^{m} \oplus \mathbb{C}^{l-m}$, $a_{1 j}=\varrho_{n, 0}\left(a_{j}\right)$ and $a_{2 j}=\varrho_{0, n}\left(a_{j}\right)$, i.e. $\varphi_{i}\left(a_{i 1}\right)=\varphi_{i}\left(a_{i 2}\right)$. Choose every $\alpha_{i}$ so that it preserves $a_{i 1}$ and $a_{i 2}$. By Proposition 1.10 it suffices to show that condition (\#) holds after the coordinate substitution $\alpha$, and by Remark 1.5 one needs only to check that $\varphi\left(T_{a_{1}} \Gamma\right) \cap \varphi\left(T_{a_{2}}(\Gamma)=\{0\}\right.$. Now the desired conclusion that the last equality holds after appropriate automorphism $\alpha$ follows from the fact that by [1, Theorem 4.14 and Remark 4.16] $\alpha_{i}$ can be also chosen with prescribed values of $\left(\alpha_{i}\right)_{*}$ in $S L\left(T_{a_{i j}} \mathbb{C}^{n}\right)$ for each $j$.

\section{The Proof of Theorem 0.1}

Proposition 2.1. Let $\Psi: X_{1} \rightarrow X_{2}$ be an isomorphism of closed affine algebraic subvarieties of $\mathbb{C}^{n}$ where $n=2 \operatorname{dim} X_{1}+1$. Suppose that $\varphi \in L_{n, n-1}$ is such that $\varphi\left(X_{1}\right)=\varphi\left(X_{2}\right)=: Y$ is closed in $\mathbb{C}^{n}$ and $\left.\varphi\right|_{X_{i}}: X_{i} \rightarrow Y$ is a semi-isomorphism for $i=1,2$. Then there exists an extension of $\Psi$ to a holomorphic automorphism $\tilde{\Psi}: \mathbb{C}^{n} \rightarrow \mathbb{C}^{n}$.

Proof. Let $\bar{z}=\left(z_{1}, \ldots, z_{n}\right)$ be a coordinate system on $\mathbb{C}^{n}$. Without loss of generality one can suppose that the coordinate form of $\varphi$ is $\bar{z} \rightarrow\left(z_{1}, \ldots, z_{n-1}\right)=: \bar{\zeta}$. Denote by $f_{i}$ the restriction of the function $z_{n}$ to $X_{i}$. Then $f_{i}$ can be treated as a rational function on $Y$ with possible indeterminacy points in $Y_{\text {sing }} \backslash S$ where $S=\varphi\left(\left(X_{1}\right)_{\text {sing }}\right)=\varphi\left(\left(X_{2}\right)_{\text {sing }}\right)$ (the last equality holds since otherwise $\Psi$ is not an isomorphism). Consider a holomorphic automorphism $\alpha$ of $\mathbb{C}^{n}$ given by $\bar{z} \rightarrow$ $\left(\bar{\zeta}, e^{g(\bar{\zeta})} z_{n}\right)$ where $g$ is a polynomial in $\bar{\zeta}$. Our aim is to choose $g$ so that $h:=$ $f_{1}-e^{g(\bar{\zeta})} f_{2}$ is a holomorphic function on $Y$.

Let $y \in Y_{\text {sing }} \backslash S$ and $\varphi^{-1}(y) \cap X_{i}=\left\{x_{i}^{\prime}, x_{i}^{\prime \prime}\right\}$. Suppose that $a_{i}^{\prime}=f_{i}\left(x_{i}^{\prime}\right)$ and $a_{i}^{\prime \prime}=f_{i}\left(x_{i}^{\prime \prime}\right)$. Note that $a_{i}^{\prime} \neq a_{i}^{\prime \prime}$ since otherwise $x_{i}^{\prime}=x_{i}^{\prime \prime}$ is a singular point of $X_{i}$ contrary to the assumption. Choose $g$ so that

$$
e^{g(y)}=\frac{a_{1}^{\prime}-a_{1}^{\prime \prime}}{a_{2}^{\prime}-a_{2}^{\prime \prime}}
$$

for every $y \in Y_{\text {sing }} \backslash S$. Then $h\left(x_{1}^{\prime}\right)=h\left(x_{1}^{\prime \prime}\right)$ and therefore $h$ can by viewed as a holomorphic function on $Y$ by Proposition 1.2 We denote by the same symbol a holomorphic extension of $h$ to $\mathbb{C}^{n-1}$. Let $\beta$ be the holomorphic automorphism of $\mathbb{C}^{n}$ given by $\bar{z} \rightarrow\left(\bar{\zeta}, z_{n}+h(\bar{\zeta})\right)$. Then the composition $\alpha^{-1} \circ \beta$ is the desired extension of $\Psi$.

Remark 2.2. (1) The same argument shows that an extension to a holomorphic automorphism in Proposition 2.1 exists in the case when $\Psi: X_{1} \rightarrow X_{2}$ is a biholomorphic map of closed analytic subsets of $\mathbb{C}^{n}$ and $Y$ is a closed analytic subset of $\mathbb{C}^{n-1}$.

(2) If $\varphi$ is only a pseudo-isomorphism, then a priori the meromorphic function $h=f_{1}-e^{g(\zeta)} f_{2}=f_{1}-f_{2}+\left(1-e^{g}(\zeta)\right) f_{2}$ constructed in the proof is holomorphic only on $Y \backslash \varphi\left(X_{\text {sing }}\right)$. However, suppose that $X_{\text {sing }}$ is, say, discrete and for every $x \in X_{\text {sing }}$ there exists a natural $k=k(x)$ such that every germ of a continuous meromorphic function, that vanishes at $x$ with multiplicity at least $k$, is in fact holomorphic at $x$. If it is also known that $f_{1}-f_{2}$ is holomorphic at points of $\varphi\left(X_{\text {sing }}\right) \subset Y$, then 
requiring additionally that $g$ vanishes on $\varphi\left(X_{\text {sing }}\right)$ with sufficiently large multiplicity, one gets $h$ holomorphic on the entire $Y$. In particular in this case, an extension of $\Psi$ to a holomorphic automorphism again exists.

Definition 2.3. Let Notation 1.11 hold but we allow $n \geq 2 \operatorname{dim} X_{1}+1$. We say that the triple $\left(\Psi, X_{1}, X_{2}\right)$ is admissible with respect to the coordinate system $(\bar{z}, \bar{w})$ if

(i) $n>l:=\max \left(2 \operatorname{dim} X_{1}, \operatorname{dim} T X_{1}\right)$;

(ii) for every $m=0,1, \ldots, l$ the set $Z_{m}=\varrho_{m, l-m}(\Gamma)$ is a closed affine algebraic subvariety of $\mathbb{C}^{l}$;

(iii) the restriction $\left.\varrho_{m, l-m}\right|_{\Gamma}: \Gamma \rightarrow Z_{m}$ is a semi-isomorphism for $m=0, \ldots, l$;

(iv) the restriction $\left.\varrho_{m, l+1-m}\right|_{\Gamma}: \Gamma \rightarrow W_{m}:=\varrho_{m, l+1-m}(\Gamma)$ is an isomorphism for $m=0, \ldots, l+1$.

Proposition 2.4. Let a triple $\left(\Psi, X_{1}, X_{2}\right)$ be admissible. Then $\Psi$ can be extended to a holomorphic automorphism $\tilde{\Psi}: \mathbb{C}^{n} \rightarrow \mathbb{C}^{n}$.

Proof. If $\operatorname{dim} T X_{1} \geq 2 \operatorname{dim} X_{1}+1$, then $\tilde{\Psi}$ can be chosen algebraic by [8]. Thus we suppose that $\operatorname{dim} T X_{1} \leq 2 \operatorname{dim} X_{1}$. By the same reason we can suppose that $n=$ $2 \operatorname{dim} X_{1}+1=l+1$. Consider $\mathbb{C}^{n}$ equipped with a coordinate system $\left(u_{1}, \ldots, u_{n}\right)$. Let $W_{m}^{\prime}$ be the image of $\Gamma$ under the morphism $\mathbb{C}^{2 n} \rightarrow \mathbb{C}^{n}$ given by

$$
(\bar{z}, \bar{w}) \rightarrow\left(z_{1}, \ldots, z_{m-1}, w_{1}, \ldots, w_{n-m}, z_{m}\right) .
$$

Note that all $W_{m}$ are closed subvarieties of $\mathbb{C}^{n}$ by condition (ii) of Definition 2.3 . The same is true for $W_{m}^{\prime}$ since $W_{m}$ and $W_{m}^{\prime}$ are isomorphic under the automorphism $\beta_{m}: \mathbb{C}^{n} \rightarrow \mathbb{C}^{n}$ that switches coordinates $u_{m}$ and $u_{n}$. Note also that $W_{0}=X_{2}$ while $W_{n}=X_{1}$. By condition (iv) of Definition 2.3 there are isomorphisms $\psi_{m}: W_{m-1} \rightarrow$ $W_{m}$ such that $\Psi=\psi_{1} \circ \cdots \circ \psi_{n}$. Consider the projection $\varphi: \mathbb{C}^{n} \rightarrow \mathbb{C}^{l}$ given by $\left(u_{1}, \ldots, u_{n}\right) \rightarrow\left(u_{1}, \ldots, u_{l}\right)$. Note that $\varphi\left(W_{m-1}\right)=\varphi\left(W_{m}^{\prime}\right)$ and by condition (iii) of Definition 2.3 the restrictions of $\varphi$ to $W_{m}$ or to $W_{m}^{\prime}$ yield semi-isomorphisms. By Proposition 2.1 there is a holomorphic automorphism $\alpha_{m}: \mathbb{C}^{n} \rightarrow \mathbb{C}^{n}$ that extends the isomorphism $\beta_{m} \circ \psi_{m}$. Hence $\beta_{m}^{-1} \circ \alpha_{m}: \mathbb{C}^{n} \rightarrow \mathbb{C}^{n}$ is the extension of $\psi_{m}$ and $\left(\beta_{1}^{-1} \circ \alpha_{1}\right) \circ \cdots \circ\left(\beta_{n}^{-1} \circ \alpha_{n}\right)$ is the desired extension of $\Psi$.

Proof of Theorem 0.1. By [8, Theorem 1] we can suppose that $\operatorname{dim} T X_{1} \leq 2 \operatorname{dim} X_{1}$. Let $l=2 \operatorname{dim} X_{1}+1$. Since for a general $\varphi \in L_{n, l}$ the restrictions $\left.\varphi\right|_{X_{i}}: X_{i} \rightarrow$ $\varphi\left(X_{i}\right), i=1,2$, are isomorphisms onto closed subvarieties of $\mathbb{C}^{l}$, we can suppose that $n=2 \operatorname{dim} X_{1}+1$. Applying algebraic automorphisms $\alpha_{i}$ of $\mathbb{C}^{n}$ to $X_{i}, i=1,2$, we can suppose that the last statement of Proposition 1.12 holds. In particular, the triple $\left(\Psi, X_{1}, X_{2}\right)$ is admissible. Hence by Proposition $2.4 \Psi$ can be extended to a holomorphic automorphism $\tilde{\Psi}$ of $\mathbb{C}^{n}$ which is the desired conclusion.

\section{THE CASE OF CURVES}

It is interesting to find out whether the restriction on $\operatorname{dim} T X_{1}$ in Theorem 0.1 can be made weaker; say, whether in the case of isolated singularities the inequality $n \geq 2 \operatorname{dim} X_{1}+1$ yields the existence of an extension to a holomorphic automorphism. At present we do not know a complete answer except for the one-dimensional case. Recall that there are isomorphic curves in $\mathbb{C}^{3}$ whose isomorphism does not extend to an algebraic automorphism 4 of $\mathbb{C}^{3}$ [8, Example] but we shall show that an extension to a holomorphic automorphism always exists.

\footnotetext{
${ }^{4}$ An example of such curves is given by the images of the morphisms $\varrho_{i}: \mathbb{C} \rightarrow \mathbb{C}^{3}$, where $\varrho_{1}(t)=\left(t^{7}, t^{11}, t^{13}\right)$ and $\varrho_{2}(t)=\left(t^{7+14}, t^{11}, t^{13}\right)$ with an isomorphism between them given by
} 
Convention 3.1. We study below affine algebraic subvarieties of $\mathbb{C}^{n}$ equipped with coordinates $(x, y, \bar{z})$ where $\bar{z}=\left(z_{1}, \ldots, z_{n-2}\right)$. For the sake of notation but without loss of generality we suppose further that $n=3$ and $(x, y, z)$ is a coordinate system on $\mathbb{C}^{3}=\mathbb{C}^{n}$.

We shall need later holomorphic automorphisms of $\mathbb{C}^{n}$ preserving some coordinates and having prescribed jets at a finite collection of points 5

Proposition 3.2. Let $S=\left\{s_{1}, \ldots, s_{k}\right\}$ be a finite subset in $\mathbb{C}^{3}$ with coordinates $(x, y, z)$ and $s_{i}=\left(x_{i}, y_{i}, z_{i}\right)$. Suppose that $z_{i} \neq z_{j}$ for $i \neq j$. Then for every natural number $m \geq 1$ and a collection of polynomials $f_{i}(x, y, z), i=1, \ldots, k$, with $f_{i}(0,0,0)=0$ there exists a holomorphic automorphism $\alpha$ of $\mathbb{C}^{3}$ identical on $S$ and such that for every $i=1, \ldots, k$ the Taylor series of $\alpha$ at $s_{i}$ is of form

$$
\begin{aligned}
\alpha(x, y, z)=(x, y, z)+\left(c_{i} \Delta x_{i}\right. & +f_{i}\left(\Delta x_{i}, \Delta y_{i}, \Delta z_{i}\right) \\
& \left.+M^{m}\left(\Delta x_{i}, \Delta y_{i}, \Delta z_{i}\right), M^{m}\left(\Delta x_{i}, \Delta y_{i}, \Delta z_{i}\right), \overline{0}\right)
\end{aligned}
$$

where $M$ is the maximal ideal of $\mathbb{C}[x, y, z]$ corresponding to the origin, $c_{i} \in \mathbb{C}^{*}$, $\Delta x_{i}=x-x_{i}, \Delta y_{i}=y-y_{i}$, and $\Delta z_{i}=z-z_{i}$.

Proof. Consider a polynomial $h(y, z)$ such that $h\left(y_{i}, z_{i}\right)=\ln c_{i}$ and $h\left(\Delta y_{i}, \Delta z_{i}\right)-$ $\ln c_{i} \in M^{m}\left(0, \Delta y_{i}, \Delta z_{i}\right)$ for every $i=1, \ldots, k$. Then using composition with the holomorphic automorphism $(x, y, z) \rightarrow\left(e^{h(y, z)} x, y, z\right)$ one can always suppose that each $c_{i}=1$. Furthermore, applying a triangular automorphism of the form $(x, y, z) \rightarrow(x+p(y, z), y, z)$ we can suppose that each $f_{i}(x, y, z)$ is divisible by $x$. Another simplifying observation is that it suffices to prove the statement in the case of $f_{i}(x, y, z)=x^{l} g(y, z)$ where $1 \leq l \leq m$. Indeed, if this is true then taking the composition of such holomorphic automorphisms with $l$ changing from 1 to $m$ one gets (1) in the general case.

Let $q_{l, m}$ be the sum of the first $m+1$ terms of the Taylor series for $1 /\left(1+l x^{l-1}\right)$ at the origin, i.e. the Jacobian of the map $(x, y) \rightarrow\left(x+x^{l}, y\left(1+q_{l, m}(x)\right)\right)$ is $1+M^{m}(x, y, 0)$. By [1, Theorem 4.14] there exists a polynomial automorphism $\beta_{0}=\left(\beta_{0}^{\prime}, \beta_{0}^{\prime \prime}\right): \mathbb{C}^{2} \rightarrow \mathbb{C}^{2}$ whose $m$-jet at the origin coincides with $(x, y) \rightarrow$ $\left(x+x^{l}, y\left(1+q_{l, m}(x)\right)\right)$. Let $r_{1}(z)$ and $r_{2}(z)$ be polynomials such that $r_{1}\left(z_{i}\right)=x_{i}$ and $r_{2}\left(z_{i}\right)=y_{i}$ for each $i$. Consider the automorphism $\beta$ of $\mathbb{C}^{3}$ given by

$$
\beta(x, y, z)=\left(r_{1}(z)+\beta_{0}^{\prime}\left(x-r_{1}(z)\right), r_{2}(z)+\beta_{0}^{\prime \prime}\left(y-r_{2}(z)\right), z\right) .
$$

Let $q(x)$ be the sum of the first $m$ terms of the Taylor series of $\ln q_{l, m}$ at the origin. Note that the composition $\beta_{1}$ of $\beta$ with the holomorphic automorphism $(x, y, z) \rightarrow\left(x, e^{-q\left(x-r_{1}(z)\right)} y, z\right)$ satisfies (1) with $f_{i}(x, y, z)=x^{l}$ for each $i$. Let $g(y, z)$ be a polynomial such that $g\left(y_{i}, z_{i}\right)=0$ for every $i$ and the Taylor series of $g$ at $\left(y_{i}, z_{i}\right)$ is of the form $g_{i}\left(y-y_{i}, z-z_{i}\right)+M^{m}\left(0, y-y_{i}, z-z_{i}\right)$ where $\left\{g_{i} \mid i=\right.$ $1, \ldots, k\}$ is a given collection of polynomials. Then the composition $\gamma_{l}$ of $\beta_{1}$ with the holomorphic automorphism $(x, y, z) \rightarrow\left(e^{g(y, z)} x, y, z\right)$ satisfies $(1)$ with $f_{i}(x, y, z)=$ $x^{l} g_{i}(y, z), i=1, \ldots, k$, and we are done.

$(x, y, z) \rightarrow\left(x+x^{2}, y, z\right)$. Actually, the argument in [8] shows that there is no extension of this isomorphism to a holomorphic automorphism with constant Jacobian.

${ }^{5}$ Holomorphic automorphisms with prescribed jets at a finite set were constructed in 5 but the condition on preservation of coordinates does not allow a direct use of that result. 
Lemma 3.3. Let $X$ and $Y$ be the germs of algebraic curves at the origin o of $\mathbb{C}^{3}$ and let $M$ be as in Proposition 3.2 . Suppose that $\psi: X \rightarrow Y$ is a bijective morphism. Then there exists a natural $m$ such that $\left.M^{m}\right|_{X} \subset \psi^{*}(\mathcal{O}(Y))$.

Proof. Suppose first that $Y$ is irreducible, i.e. it is the image of the germ $(\mathbb{C}, 0)$ under an injective holomorphic map $\lambda:(\mathbb{C}, 0) \rightarrow(Y, o)$. Let $R=\lambda^{*}(\mathcal{O}(Y))$ and let $S$ be the similar ring for $X$, i.e. $S$ can be viewed as a finitely generated $R$-module containing $R$ as a submodule. The set of zero multiplicities of functions from $R$ at $0 \in \mathbb{C}$ form a semi-group $G$ and, furthermore, there exists a natural $m$ such that $G$ contains $\{n \in \mathbb{N} \mid n \geq m\}$ 盀

Suppose that $I$ is the ideal of $S$ induced by $\left.M^{m}\right|_{X}$. By the Krull theorem (e.g., see [3]) $\bigcap_{k=1}^{\infty} I^{k}=0$. Hence $\bigcap_{k=1}^{\infty} I^{k} /(R \cap I)=0$. Then the above property of the semi-group $G$ implies that for every $k>0$ and $f \in I$ there exists $g \in R \cap I$ such that $f+g \in I^{k}$. That is, the image of $f$ in $I /(R \cap I)$ is zero which implies the desired conclusion in the irreducible case.

Suppose now that $Y$ is not irreducible. Say it consists of two components $Y^{\prime}$ and $Y^{\prime \prime}$. Let $m^{\prime}$ and $m^{\prime \prime}$ play the same role for this component as $m$ for $Y$ in the irreducible case. Let $g^{\prime}$ be a holomorphic function that vanishes on $Y^{\prime \prime}$ but not on $Y^{\prime}$ while $g^{\prime \prime}$ plays the opposite role. Let $n^{\prime}$ be the zero multiplicity of $g^{\prime}$ on $Y^{\prime}$ and $n^{\prime \prime}$ the zero multiplicity of $g^{\prime \prime}$ on $Y^{\prime \prime}$. The irreducible case implies now that any function on $Y$ that vanishes on $Y^{\prime \prime}$ (resp. $Y^{\prime}$ ) and has zero multiplicity $n^{\prime}+m^{\prime}$ on $Y^{\prime}$ (resp. $n^{\prime \prime}+m^{\prime \prime}$ on $Y^{\prime \prime}$ ) is contained in $R$. Taking the sum of such functions we get the desired conclusion with $m=\max \left(n^{\prime}+m^{\prime}, n^{\prime \prime}+m^{\prime \prime}\right)$.

Proposition 3.4. Let $\varphi: \mathbb{C}^{3} \rightarrow \mathbb{C}_{y, z}^{2}$ be the forgetting projection, let $\Psi: X_{1} \rightarrow X_{2}$ be an isomorphism of closed affine algebraic curves in $\mathbb{C}^{3}$ such that $\left.\varphi\right|_{X_{1}}=\varphi \circ \Psi$, and let $\left.\varphi\right|_{X_{i}}: X_{i} \rightarrow Y:=\varphi\left(X_{i}\right)$ be a pseudo-isomorphism. Suppose also that we have $\left(X_{1}\right)_{\text {sing }}=\left(X_{2}\right)_{\operatorname{sing}}=: S$. Then there exists an extension of $\Psi$ to a holomorphic automorphism of $\mathbb{C}^{3}$.

Proof. By assumption the coordinate form of $\Psi$ is $(x, y, z) \rightarrow(F(x, y, z), y, z)$. Making a linear substitution in $\mathbb{C}_{y, z}^{2}$ one can suppose that the restriction of the forgetting projection $\mathbb{C}^{3} \rightarrow \mathbb{C}_{z}$ to $S:=\left(X_{i}\right)_{\text {sing }}$ is injective and since $\Psi$ is an isomorphism at every $s_{i}=\left(x_{i}, y_{i}, z_{i}\right) \in S$ the Taylor series of $F$ is of the form $F(x, y, z)=c_{i} x_{i}+f_{i}\left(\Delta x_{i}, \Delta y_{i}, \Delta z_{i}\right)+M^{m}\left(\Delta x_{i}, \Delta y_{i}, \Delta z_{i}\right)$ where $m$ is a sufficiently large natural number, and $c_{i}, f_{i}, \Delta x_{i}, \Delta y_{i}, \Delta z_{i}$, and $M$ are as in Proposition 3.2 . Hence applying a holomorphic automorphism as in Proposition 3.2 to $X_{1}$ we can suppose that $c_{i}=1$ and $f_{i} \equiv 0$ for every $s_{i} \in S$. Consider $h=\left.x\right|_{X_{1}}-\left.x\right|_{X_{2}}$ as a meromorphic function on $Y$. Note that by Lemma $3.3 h$ is holomorphic at points $\varphi(S)$. Now the desired conclusion follows from Remark $2.2(2)$.

Proof of Theorem 0.2. By Convention 3.1 we consider the case of curves in $\mathbb{C}^{3}$. Applying a polynomial automorphism to $X_{2}$ one can suppose that $S:=\left(X_{1}\right)_{\text {sing }}$

\footnotetext{
${ }^{6}$ Since the author does not know a reference to this well-known fact we sketch a proof. Let $k \in \mathbb{N}$ be the greatest common divisor of elements of $G$, i.e. for some $m>0$ this semi-group contains $\{n k \mid n \in \mathbb{N}, n \geq m / k\}$. Assume that $k \geq 2$. There must be a function $f \in \mathcal{O}(Y)$ such that the Taylor series $f \circ \lambda(t)=\sum_{i=1}^{\infty} a_{i} t^{i}$ contains a non-zero term $a_{j} t^{j}$ with $i$ non-divisible by $k$ since otherwise the map $\lambda$ is not injective. Replacing $f$ with its power one can suppose that $a_{1}=\ldots=a_{m}=0$ and still have some $a_{i} \neq 0$ with $i$ non-divisible by $k$. Hence adding to $f$ an element of $R$ we can get a function from $R$ whose zero multiplicity is not divisible by $k$. A contradiction.
} 
coincides with $\left(X_{2}\right)_{\text {sing }}$ as a subset of $\mathbb{C}^{n}$. Furthermore, by Proposition 1.12 applying polynomial automorphisms to $X_{i}$ we can suppose that the restriction of any forgetting coordinate projection $p: \mathbb{C}_{x_{1}, y_{1}, z_{1}, x_{2}, y_{2}, z_{2}}^{6} \rightarrow \mathbb{C}^{3}$ to the graph $\Gamma$ of $\Psi$ yields an isomorphism between $\Gamma$ and its image. By virtue of Remark 1.5 (2) one can suppose that these automorphisms do not ruin the equality $\left(X_{1}\right)_{\operatorname{sing}}=\left(X_{2}\right)_{\text {sing }}$ since they can be chosen identical on $S$. Furthermore, applying a linear automorphism to both $X_{1}$ and $X_{2}$ we get the following:

(i) the restriction of any coordinate to $S$ is injective;

(ii) the restriction of any coordinate to $X_{i}, i=1,2$, is a proper map onto $\mathbb{C}$,

(iii) for any forgetting coordinate projection $\mathbb{C}^{6} \rightarrow \mathbb{C}^{2}$ its restriction to each $X_{i}$ yields a pseudo-isomorphism.

Consider the image $X_{3}$ (resp. $X_{4}$ ) of $\Gamma$ under the projection $\mathbb{C}^{6} \rightarrow \mathbb{C}^{3}$ given by $\left(x_{1}, y_{1}, z_{1}, x_{2}, y_{2}, z_{2}\right) \rightarrow\left(x_{2}, y_{1}, z_{1}\right)$ (resp. $\left.\left(x_{1}, y_{1}, z_{1}, x_{2}, y_{2}, z_{2}\right) \rightarrow\left(x_{2}, y_{2}, z_{1}\right)\right)$. Suppose that $X_{3}$ and $X_{4}$ are naturally embedded in the same sample of $\mathbb{C}^{3}$ as $X_{1}$ and $X_{2}$. By Proposition 3.4 the natural isomorphism $X_{1} \rightarrow X_{3}$ over $\mathbb{C}_{y_{1}, z_{1}}^{2}$ (resp. $X_{3} \rightarrow X_{4}$ over $\mathbb{C}_{x_{2}, z_{1}}^{2}$; resp. $X_{4} \rightarrow X_{2}$ over $\mathbb{C}_{x_{2}, y_{2}}^{2}$ ) extends to a holomorphic automorphism of $\mathbb{C}^{3}$. Taking composition of these holomorphic automorphisms we get the desired conclusion.

Remark 3.5. We used only two specific properties of curves in the proof of Theorem 0.2. Namely, that

(1) the set $S$ of singularities of $X_{i} \subset \mathbb{C}^{n}$, whose Zariski tangent space is of dimension $n$, is finite and the dimension of $T(X \backslash S)$ is at most $n-1$;

(2) for every $x \in S$ each continuous meromorphic function vanishing at $x$ with sufficiently large multiplicity (independent on the function) is in fact holomorphic at $x$.

Hence the same argument leads to a more general statement.

Let $\Psi: X_{1} \rightarrow X_{2}$ be an isomorphism of closed affine subvarieties of $\mathbb{C}^{n}$ such that the singularities of $X_{1}$ satisfy conditions (1) and (2) above. Suppose also that $n \geq 2 \operatorname{dim} X+1$. Then $\Psi$ extends to a holomorphic automorphism of $\mathbb{C}^{n}$.

\section{ACKNOWLEDGMENT}

We are grateful to G. Freudenburg who shared with the author some of his unpublished results on algebraic extensions of curve isomorphisms in $\mathbb{C}^{3}$ and whose questions stimulated this study.

\section{REFERENCES}

[1] I. Arzhantsev, H. Flenner, S. Kaliman, F. Kutzschebauch, and M. Zaidenberg, Flexible varieties and automorphism groups, Duke Math. J. 162 (2013), no. 4, 767-823, DOI 10.1215/00127094-2080132. MR.3039680

[2] Harm Derksen, Frank Kutzschebauch, and Jörg Winkelmann, Subvarieties of $\mathbf{C}^{n}$ with non-extendable automorphisms, J. Reine Angew. Math. 508 (1999), 213-235, DOI 10.1515/crll.1999.028. MR 1676877 (2000c:32072)

[3] David Eisenbud, Commutative algebra, Graduate Texts in Mathematics, vol. 150, SpringerVerlag, New York, 1995. With a view toward algebraic geometry. MR1322960 (97a:13001)

[4] H. Flenner, S. Kaliman, M. Zaidenberg, A Gromov-Winkelmann type theorem for flexible varieites, arXiv:1305.6417, MPI preprint 2013-30, (to appear in J. of Eur. Math. Soc.).

[5] Franc Forstnerič, Holomorphic flexibility properties of complex manifolds, Amer. J. Math. 128 (2006), no. 1, 239-270. MR2197073 (2006k:32024)

[6] Franc Forstneric, Josip Globevnik, and Jean-Pierre Rosay, Nonstraightenable complex lines in $\mathbf{C}^{2}$, Ark. Mat. 34 (1996), no. 1, 97-101, DOI 10.1007/BF02559509. MR.1396625(97b:32033) 
[7] Robin Hartshorne, Algebraic geometry, Springer-Verlag, New York-Heidelberg, 1977. Graduate Texts in Mathematics, No. 52. MR0463157 (57 \#3116)

[8] Shulim Kaliman, Extensions of isomorphisms between affine algebraic subvarieties of $k^{n}$ to automorphisms of $k^{n}$, Proc. Amer. Math. Soc. 113 (1991), no. 2, 325-334, DOI 10.2307/2048516. MR 1076575 (91m:14019)

[9] Shulim Kaliman, Isotopic embeddings of affine algebraic varieties into $\mathbf{C}^{n}$, The Madison Symposium on Complex Analysis (Madison, WI, 1991), Contemp. Math., vol. 137, Amer. Math. Soc., Providence, RI, 1992, pp. 291-295, DOI 10.1090/conm/137/1190990. MR1190990 (94a:14011)

[10] V. Srinivas, On the embedding dimension of an affine variety, Math. Ann. 289 (1991), no. 1, 125-132, DOI 10.1007/BF01446563. MR1087241 (92a:14013)

Department of Mathematics, University of Miami, Coral Gables, Florida 33124

E-mail address: kaliman@math.miami.edu 